\title{
VISUAL-AUDITORY VOLUME RENDERING OF SCALAR FIELDS
}

\author{
E. Malikova ${ }^{1}$, V. Adzhiev ${ }^{1}$, O. Fryazinov ${ }^{1}$, A. Pasko $^{2}$ \\ ${ }^{1}$ Bournemouth University,UK \\ ${ }^{2}$ Skolkovo Institute of Science and Technology, Moscow, Russia \\ emalikova@bournemouth.ac.uk, vadzhiev@bournemouth.ac.uk, \\ ofryazinov@bournemouth.ac.uk, apasko@bournemouth.ac.uk
}

\begin{abstract}
This paper describes a novel approach to visual-auditory volume rendering of continuous scalar fields. The proposed method uses well-established similarities in light transfer and sound propagation modelling to extend the visual scalar field data analysis with auditory attributes. We address the visual perception limitations of existing volume rendering techniques and show that they can be handled by auditory analysis. In particular, we describe a practical application to demonstrate how the proposed approach may keep the researcher aware of the visual perception issues in colour mapping and help track and detect geometrical features and symmetry break, issues that are important in the context of interpretation of the physical phenomena.
\end{abstract}

\section{INTRODUCTION}

The results of a numerical simulation or experimental measurements are raw and complex scientific data that contains a lot of information. Thus the scientific data can be quite difficult to understand and analyse. One of the examples of such data representation is a scalar field.

Scalar fields are used in many research areas, where computer simulations or experimental studies are involved, such as computational chemistry, medical data analysis and physical phenomena studies. The scalar field can have either discrete or continuous representation. In this work, we consider a more general case of continuous scalar fields. Visualisation of continuous scalar fields, however, is not always straightforward, especially when a complex phenomenon is represented. The examples of such situations are the simultaneous analysis of several scalar fields with different field features and underlying processes; scalar fields after postprocessing with image processing applied to 3D textures; application of various optical models in the visualisation pipeline. The main aim of those procedures is to highlight features of interest [1], enhance visual analysis quality [2] and handle image quality issues, arising due to limitations of scanning devices and human perception. Without these techniques, we may get a wrong insight on data, which fails the entire analysis process [3].

Visualisation of scalar fields usually employs Volume Rendering techniques as for the computer systems the scalar fields data is often converted to data volumes stored in the texture memory

(c) (1) (8) This work is licensed under Creative Commons Attribution Non Commercial 4.0 International License. The full terms of the License are available at http://creativecommons.org/licenses/by-nc/4.0
[4, 5]. In the Volume Rendering the enhancement of optical model is used to address the issues of visual analysis quality improvement $[6,7]$. The most recently introduced techniques, such as multidimensional transfer functions, are relatively new and as visualisation tools are the areas of active research [8].

The conventional Volume Rendering techniques can fail as important details and features might be missed, especially when they are relatively small. Moreover, the problem becomes even more apparent for visualisation of the dynamic objects, as it becomes hard to track small, visually hard to distinguish scalar field feature changes. This problem arises in various application domains, where small local changes in the field surfaces should be detected in order to highlight areas of potential physical properties change (e.g., superconductor fields study considered in this work). Tracking of the small changes in the dynamic scalar fields can be solved with additional numerical methods, which makes the whole process even less efficient.

It is well proven that the sensory stimuli operate differently, and thus they can successfully complement each other in the analysis process. Sonification techniques [9] as an approach to data analysis via various sound characteristics proved to be quite effective for multivariate data [10].

A visual system is limited to the perception of a certain amount of colours and shades, can be overloaded and perturbed due to fatigue. The auditory system, on the contrary, can operate in the background mode and act as an early alarm tracking even small changes via sound wave parameters. In this work, we propose a general approach to the visual-auditory analysis of scalar fields by extending the Volume Rendering technique with additional auditory stimuli. The main aim of the approach is to address visual perception issues and enhance analysis quality.

The contributions of the research are:

1. We have proposed the general model for the representation of objects with optic and auditory properties.

2. We have explored similarities between light and sound propagation to suggest a visual-auditory rendering based on the well known ray-marching procedure.

3. We have considered situations when volume rendering is insufficient or does not allow for enhancing a small features analysis. The introduced novel approach can be used to address those problems.

4. To demonstrate how the introduced method works in practice, we have applied it to enhance the quality of visual analysis while detecting small changes in the symmetry of the superconductor field. 
5. We have implemented the basic prototype of the proposed approach to visual-audio rendering. We discuss the application limitations and possibilities of the proposed approach to visual-auditory analysis.

The structure of the paper is as follows. The "Related works" section gives an overview of the scalar fields analysis problem by Volume Rendering technique. The "Visual-auditory volume rendering" section introduces an approach to visual-auditory analysis and specifies the details of visual-auditory rendering and visualauditory stimuli interpretation. The application case studies are presented in the "Experiments and case study" section. Future directions are presented in the "Conclusions" section.

\section{RELATED WORKS}

In this work, we concentrate on the Volume Rendering technique for scientific data visualisation. The research in this area emphasises the problems of visual analysis efficiency, perception and quality. A "good" visualisation is the one that leads to the automatic detection and extraction of the requested features and hides unnecessary details[6], [4], [5]. This task is particularly difficult for experimental data as it is obtained with scanning and measuring devices. The device limitations and scans quality issues inevitably arise during the process. The problem is addressed with additional procedures and techniques [11],[12].

Currently, the Direct Volume Rendering technique is used to address issues like enhancement of visual image quality, making images more perceivable and analysis more stable. The relatively new technique is Multidimensional Transfer Functions (TF) $[13,6]$. The Multidimensional Transfer Functions design is closely related to techniques and approaches used in image processing $[14,15]$ in order to introduce a more enhanced optical model to facilitate the visual analysis.

However, the visual analysis has perception limitations that cannot be addressed solely by enhancement of the optical model. The introduction of the other sensory stimuli can significantly enhance the analysis process. The research in this area $[10,16]$ stresses the visual perception of temporal and spatial resolution limitations. On the contrary, the sound wave most perceptionally efficient parameters are $1000-4000 \mathrm{~Hz}$ frequency and $0-160 \mathrm{Db}$ loudness. The use of sound is a well-known solution to track small changes, operate in background mode as an early alarm system and effectiveness for classification tasks [17].

The use of sound has been widely investigated since early 80-s $[18,17]$. The fundamental works were published in 90-s [9] and theoretical research still continues [10]. The technique of data representation using various sound characteristics is called data sonification $[19,10]$. The auditory perception brings the unique possibility to distinguish small variations in the parameters of the single sound wave and to compare sound waves. The sound analysis may be efficient for fixing a visual perception [20] or a haptic perception issues [21].

The enhancement of visual analysis of continuous scalar fields with auditory tools is a relatively new area. Some general possible auditory application directions considered in [22]. The problems of visualisation uncertainty [23] and medical data analysis [24] can be identified as areas that can most benefit from sonification. The works [23] and [24] particularly address research areas, where visual analysis of scalar fields may fail. There is an increase of interest to objects sonification and continuous data recently, in

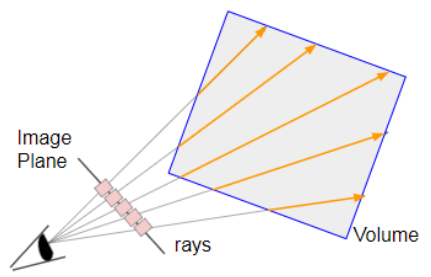

Figure 1: Backward or eye-tracing scheme in computer graphics

particular in augmented reality area [25] that takes advantage of human 3D spatial positioning of sound sources through hearing.

In this work, we introduce additional auditory sensory stimuli to address the problems arising in visual analysis. We take advantage of similarities of light and sound propagation to review the Volume Rendering technique and to extend it for a visual-auditory rendering case. We concentrate on the use of ray-tracing procedure application for computation of optical and auditory properties.

\section{VISUAL-AUDITORY VOLUME RENDERING}

\subsection{Approach overview}

Scalar field is a function $f(X): X \rightarrow \Re, X \in \Re^{n}$, which associates any point in space with a scalar value. In computer graphics, scalar fields are often used to represent geometry in an implicit form. In our work, we discuss the generic scalar fields which can represent any type of scientific data. Scalar fields are usually represented in the computer systems as scalar values stored as multi-dimensional data volumes inside the texture memory and are conveniently visualised with Volume Rendering methods.

The core of the conventional Volume Rendering technique is an emission-absorption optical model. The Volume Rendering equation is derived from the rendering equation [26] that is a fundamental concept in computer graphics on how the general optical material properties can be described through physics process of light interaction with the object if the wave nature of light can be neglected [27].The conventional Volume Rendering equation 1 takes advantage of a simplified model of light interaction with an object, that considers only emission and absorption mechanisms [28].

$$
I(D)=I_{0} e^{-\int_{s_{0}}^{D} \tau(t) d t}+\int_{s_{0}}^{D} q(s) e^{-\int_{s}^{D} \tau(t) d t} d s
$$

where I is light traversing from entry point to volume $s_{0}$ to exit point towards the camera $s=D$ intensity,

$q(s)$ is a light contribution at point s, it other words term describing emission process,

$\tau(t)$ is used to describe light attenuation, when it reaches point $\mathrm{D}$, in other words, term describing absorption process.

The concept of shooting rays is used to compute the final image that researchers see (see Fig. 1). The ray-casting or raymarching volume technique [29] solves the equation 1 by approximating the light propagation and interaction with each of volume elements along ray path.

The ray-casting procedure operates a Transfer Function (TF) defining contribution of each volume element (see Fig. 2). In this 
work, we consider the Front-to-Back Compositing scheme [30] as a numerical solution of equation 1 for each ray that is shoot from camera (see Fig. 1).

The core idea of our method is that the principles of light and sound propagation are very similar [31]. The acoustic rendering equation [32] is a time-dependent version of the same rendering equation [26]. In this work, we stress the following core similarities and differences in optical and acoustic properties modelling. First, the role of the propagation procedure that allows us to consider the ray-tracing technique as a core component of both models. The successful application of the ray-based model for sound propagation modelling demonstrates that to some extent the wave nature can be neglected. Second, the concept of radiance of the amount of energy transmitted as the result of interaction at a specific point can be used for both optical and acoustic properties modelling[32]. Finally, as follows from the acoustic rendering equation, the main difference between light and sound propagation modelling is that the aural perception of the time/passed distance dependency for the sound [32].

We take advantage of similarities in modelling optic and acoustic properties of objects due to the similar nature of propagation of light and sound and propose an acoustic model that describes the acoustic properties of the object. The model is based on a conventional Volume Rendering optic model and considers timedependent emission and absorption processes of a sound wave propagation

The basic concept of the proposed auditory model of sound wave generation as a result of an impulse propagation through the object is shared by both ray-based and wave-based approaches to sound modelling, like digital waveguide and the banded digital waveguide approaches to physically based sound synthesis [33]. These approaches establish relation between sound propagation and modal sound synthesis [33]. The sound wave is modelled as a result of a propagation process, while each activated mode depends on a sound propagation path in the vibrating object and final sound is a contribution of all modes computed for considered rays.

However, in the context of the proposed auditory transfer function, the sound propagation on the basis of the wave model will lead to the possible change of the perceived sound property, namely of the pitch. Thus, the further aural perception and interpretation of such auditory model can be difficult. For this reason the conceptual framework considers wave-based approaches, but in this work does not take direct advantage of them. We will consider the extension of the emission-absorption optical model as the proposed acoustic interaction model and concentrate mainly on the ray-based approach, although, as follows from the above discussions, some parallels to physically based sound synthesis can be made. We will also take advantage of established terms by introducing the "modal areas" that are activated by the travelling ray.

\subsection{Object with optical and auditory properties}

To keep the auditory model within the concept of scalar fields, we use the idea of a HyperVolume (HV) model [34] described with an equation:

$O=\left(G, A_{o}, A_{s}\right):\left(f(X), S_{o}(X), S_{s}(X)\right)$

In the HV model, the scalar field function is augmented with point attribute functions $S$ defining optical $A_{o}$ and auditory $A_{s}$ properties. Thus, we effectively define a vector field, or vectorvalued function whose first component $f$ is responsible for the object geometry $G$ and maps directly from the input scalar field.

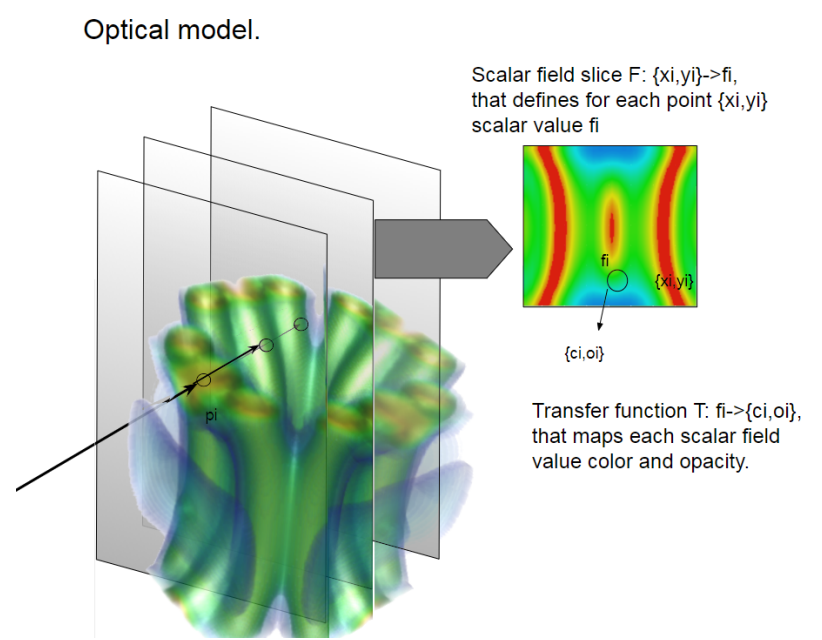

Figure 2: Volume Rendering optical model. Colour at point pi equals to the amount of light that has reached this point multiplied by emission of selected wavelengths perceived as colour. The final pixel colour is a sum of all colours in points pi along ray that is calculated with a ray-marching procedure. $S_{o}=T(F(x))$, where scalar field $F(X), T$ - transfer function.

Other components serve as the point attributes for visual and auditory properties. Auditory properties in a form of the generated sound wave are defined with $S_{s}(X)$ and the attribute $S_{o}$ define the results of mapping to the optical properties such as colour and opacity. Note that in the general case attributes $S$ are not scalars but vectors. For example, the colour information stored in $S_{t}$ is normally represented as a four-component RGB value and opacity.

The optical properties of the model $O$ can be rendered directly with a Volume Rendering technique, operating a ray-casting procedure. For the model $O$, the attribute function $S_{o}$ is a result of the Volume Rendering transfer functions that will operate the scalar field normalisation procedure to perform the mapping. The process is schematically presented in Fig. 2.

We will introduce an auditory model and will describe how the final sound can be obtained with the ray-marching procedure similarly to conventional Volume Rendering technique.

\subsection{Auditory model}

For the introduced time-dependent auditory model we consider the traditional two parts of sound modelling [31] in terms of listener perception. The first step is considering how a wave propagates through the object represented by the scalar field; the second step is exploration how a resulting sound propagates through an environment and interacts with a listener thus enabling the perception of the sound spatial properties(e.g., sound source position).

For the first part, we define an auditory transfer function to obtain a time-dependent auditory attributes $S_{s}(X(t))$ of our HV model. The auditory transfer function is designed to be operated by ray-tracing procedure that automatically produces an output allowing for efficient judgement on the considered scalar field properties along the ray. An auditory rendering procedure becomes very much similar to optic rendering procedure in a conventional 
Volume Rendering as they both consider physical processes of propagation.

We propose the researcher perceives and analyses the generated auditory properties in terms similar to how the final sound is formed in space as a result of an initial impulse (interaction). Below we describe the proposed auditory transfer function designed to address visual perception limitations as tracking of small changes in the scalar field are required.

For the second part, we consider the use of the pre-computed Head-related transfer function (HRTF) that convolves sounds generated by the object as it propagates to the left and right ears. HRTF is represented with a frequency domain of a head-related impulse response (HRIR). Application of HRTF suggests an extraction of HRIR coefficients and delays to compute sound convolution. A sound source coordinates are matched with HRTF coordinates and HRIR coefficients, and delays are interpolated via bilinear interpolation as the measurements are discrete [35]. The sound pressure for left and right ears $H_{L}$ and $H_{R}$ are defined as [35]:

$$
H_{L}=\frac{P_{L}(r, \theta, \phi, f)}{P_{0}(r, f)}, H_{R}=\frac{P_{R}(r, \theta, \phi, f)}{P_{0}(r, f)}
$$

where the sound source is defined with spherical coordinates $(r, \theta, \phi), P_{L}$ and $P_{R}$ are complex sound pressures at the entrance of left and right ears, and $P_{0}$ is a complex sound pressure at the centre of listeners head. The process is schematically presented at Fig. 3).

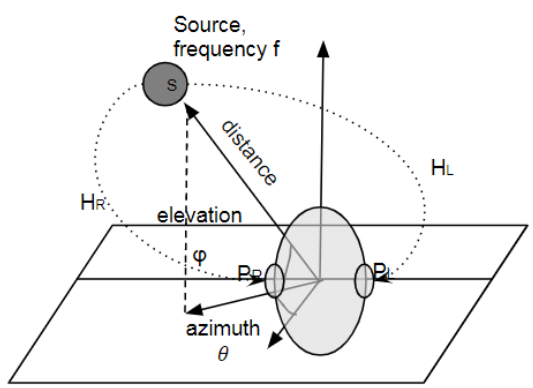

Figure 3: HRTF scheme

\subsection{Auditory rendering for tracking small changes in the scalar field}

\subsubsection{Auditory transfer function}

The main requirement to the conventional Volume Rendering transfer function [6] is to highlight features of interest via optical attributes, which normally are colour and opacity. Similarly, the auditory attributes in our model should allow the user to efficiently analyse the scalar field. Traditional auditory properties which allow for doing so are pitch, volume and sound's spatial properties. According to the modal synthesis approach [33], the sound produced by the object can be represented as a sum of the weighted modal modes $N^{\prime}$ (sound wave components with specified frequencies) extracted with Fourier Transform. However, a normal listener perceives only one or several frequencies as pitches from the entire complex sound and can interpret a sound wave in musical terms as a pitch or chord sequence. All the other components form the sound quality characteristics that allow us to aurally distinguish one musical instrument from another.

Let us consider the mapping $M: f->w$ of scalar field values $f$ to more pleasant "musical" sounds such as sequences of the specified musical pitches of frequencies $w$. In our approach, to specify these frequencies we are using MIDI (stands for Musical Instrument Digital Interface). In music, the MIDI format is widely used to formalise the sound representation, and the basic MIDI message tuple $\left(O n / O f f, M I D I_{\text {Key }}, M I D I_{\text {KeyVelocity }}\right)$ can be used to find the wave duration, the frequency and the amplitude directly. Therefore, the auditory properties, which we store in the HV model, are mapped from MIDI message components as $M: f->M I D I_{K e y}$, where field $M I D I_{K e y}$ represents frequencies and act like an auditory transfer function.

The $M I D I$ field splits scalar field to separate areas (Fig. 4) that produce a modal vibration, as the sound propagates through them. To roughly define them we introduce a term the separate object "modal area". Thus, to establish the mapping $M: f->w$, we select a musical scale with degree numbers $0, . . N$ within the specified range $N$. Whilst small range scales are easier to perceive, a bigger range gives a trained listener more options for judging about small data changes. Our experiments showed that in most cases Cmaj of up to two-octave range is sufficient to auditory highlight areas the visual analysis might miss. The mapping, therefore, is described as follows:

1. To establish the mapping $f->0, . . N$, we calculate the scale degree $n_{i} \in 0, . . N$ for each scalar filed value $f(X)$ within the sub-range as $n_{i}=\left\lfloor\frac{f(X)}{\Delta d}\right\rfloor$, where $\Delta d=$ $\frac{f_{\max }-f_{\min }}{N}$

2. $0, . . N->M I D I_{n}$. The mapping for Cmaj scale of the defined range and the start key can easily be implemented on the basis of knowledge about the major scale structure of a combination of tones (T) and semi-tone (S) intervals between notes (TTSTTTS) [36].

3. The mapping $M I D I_{\text {Key }}->w$ can be obtained with well known MIDI keynote to the frequency conversion equation.

The result of an acoustic transfer function is used by the raymarching procedure in order to generate the sound, which is perceived by the listener. The idea is to generate the final sound from the initial sound impulse as it propagates along the ray path in a scalar field through time, activating the modes with specified acoustic parameters as described below.

\subsubsection{Ray-casting procedure}

From a physics point of view, the mapping of scalar field to optical properties defines how the single field point interacts with light[30]. The introduced auditory transfer function describes how a segment of the field of a certain length (a ray-traced modal area) interacts with sound impulse that propagates through the scalar field along a defined path. Thus, the acoustic model follows the same principles and uses a similar to the optical model definition with the discrete number of modal areas and distance-dependent ray-casting procedure employed.

The conventional volume rendering equation is solved with ray-marching procedure by Front-to-Back Compositing scheme 
[30]:

$$
\begin{gathered}
\hat{C}_{i}=C_{i}\left(1-\hat{A}_{i-1}\right)+\hat{C}_{i-1} \\
\left.\hat{A}_{i}=A_{i}\left(1-\hat{A}_{i-1}\right)\right)+\hat{A}_{i-1}
\end{gathered}
$$

where $\hat{C}_{i-1}$ and $\hat{A}_{i-1}$ are the colour and opacity accumulated on previous step, and $C_{i}$ and $A_{i}$ are values that the transfer function returns for the current pixel.

Similarly, as the sound impulse propagates along the ray, the total impulse for the current modal area will be the sum of the accumulated by all previous modes impulse and of the value returned by the auditory transfer function for the current mode. As sound propagation is distance dependent, the modes are activated in time and attenuated depending on the distance passed. We adjust attenuation parameters for each mode in such a way that the produced modal oscillations do not significantly overlap. Thus it is easier to judge on the field values through the frequency of the mode. Taking advantage of a sampled digital wave representation, we represent the entire ray propagation path with a sampled buffer. As the distance for the current mode is computed, we write it into a buffer, regularly updating an output signal, while the ray-casting procedure operates the scalar field acoustic transfer function along a path.

We summarise the process in the following distance dependent equation for a sampled continuous sound wave:

$$
I(d)=\sum_{i=0}^{N} A_{i} * M_{i} * \begin{cases}0, & \text { if } D_{i}-d>0 \\ e^{d u r_{i} *\left(D_{i}-d\right)}, & \text { otherwise }\end{cases}
$$

where $d$ denotes the distance the ray passes, which is proportional to the time parameter of a spreading wave impulse; $N$ is a total number of modes with the times they are activated/intersected as the ray travels; $d u r_{i}$ is a mode duration that can be computed as difference in $D_{i}$ of the current mode and $D_{k}$ of the next mode $D_{k}=\min _{j=0}^{j=n}\left(D_{j}\right)>D_{i} . M_{i}$ is a mode described in the form of $M_{i}=\sin \left(w_{i} *\left(d-D_{i}\right)\right)$, where $D_{i}$ is a distance along the ray before intersecting a patch area of $i$ mode, $w_{i}$ is a mode frequency for the MIDI field, which is obtained from the scalar field as described above, and finally $A_{i}$ denotes the initial amplitude of the mode, which describes the energy the impulse transmits to the mode. As the ray travels, the impulse attenuates due to absorption and is described with equation $A\left(D_{i}\right)=A_{i n i} * \exp ^{-m * D_{i}}$.

Similarly to the computation of a pixel colour in Volume Rendering, the final acoustic impulse is a weighted sum of all the modes activated along the ray. The ray-marching procedure uses the modal frequency $w_{i}$ and the initial amplitude $A_{i}$ in a similar way to the optical model: the modal frequency is mapped from the source scalar field (it acts similarly to the colour attribute in the optical model), and the amplitude acts like opacity in the optical model. Consequently the duration $d u r_{i}$ appears due to the time-dependent nature of sound and is perceived through a delay of activation. The general similarities between optical and auditory models are demonstrated in Fig. 2 and Fig. 4. The similarities of the conventional optical model and the proposed auditory model make the last one convenient to address the issues, where the optical model can fail such as a colour mapping evaluation and tracking small changes in a scalar field.

At the final step, we apply the HRTF convolution to the generated sound wave to allow the listener to track the scanning ray path

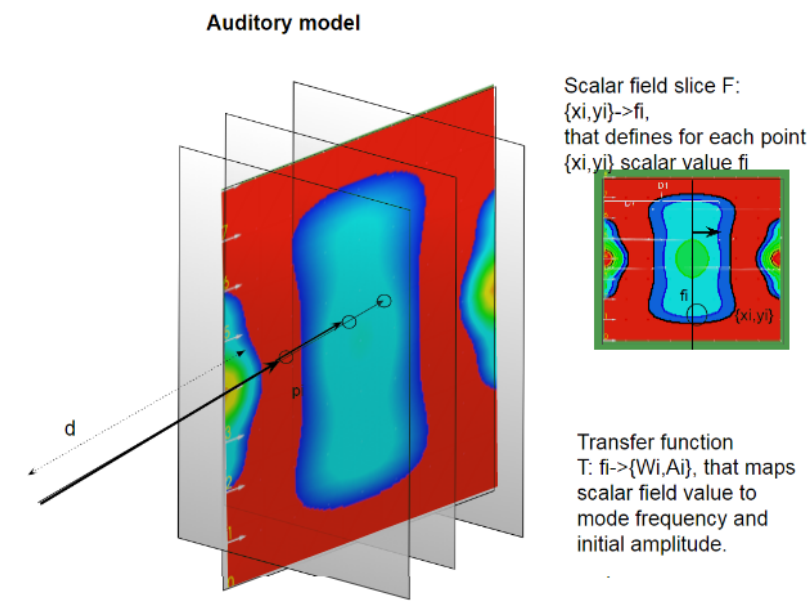

Figure 4: Introduced auditory model. The sound wave at point $p_{i}=A_{i} * \sin \left(W_{i} * d\right)$ as the impulse has propagated for distance d. $W_{i}$ controls emission part - frequencies of generated sound; $A_{i}$ controls absorption part - the amount of initial impulse energy that has reached the point. The final sound wave is a sum of all waves generated along the ray path and is calculated with a ray-marching procedure.

in space by defining the sound source position in the time equal to the current scanning position along the ray.

The introduced scanning ray procedure is a basis for the auditory analysis of the scalar field. As an acoustic impulse propagates, the user evaluates the scalar field features through the timedependent changes in the pitch. Below we will consider some case studies that demonstrate how the introduced approach to an auditory analysis can enhance the quality of the visual analysis.

\subsubsection{Interactive procedures}

Additionally, we describe an interactive data manipulation procedures on the basis of auditory information. The interaction is based on defining "musical queries" that are the sequences of notes. Similar to rendering, the interaction procedures operate with MIDI field. For the musical query simplification, we neglect all the message components except the key number that defines a note.

We demonstrate a simple example (Fig. 5 a,b and the accompanying video [37]). We use the MIDI keyboard for a fast extraction of a particular part of a field that demonstrates the musical pattern of interest (the scan is taken along the $y$-axis). The music pattern is defined with MIDI keyboard (Fig. 5 a). We can search and highlight the scalar field areas demonstrating the same pitch pattern (Fig. $5 \mathrm{~b}$ ) via the defined field $M I D I_{n}$ and thus quickly define the area of interest in a scalar field. The technique may be used for the search for a smooth/fast gradient changes detection as well

\section{A CASE STUDY OF SUPERCONDUCTOR'S FIELD}

Our approach to visual-auditory analysis has been implemented with C++/Python with using of VTK [38], OpenAL [39] and OpenCV [40] libraries. The interface procedures for the described 


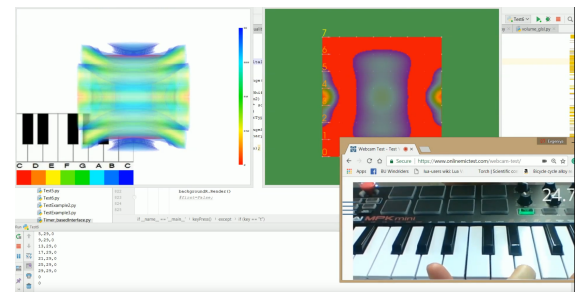

(a)

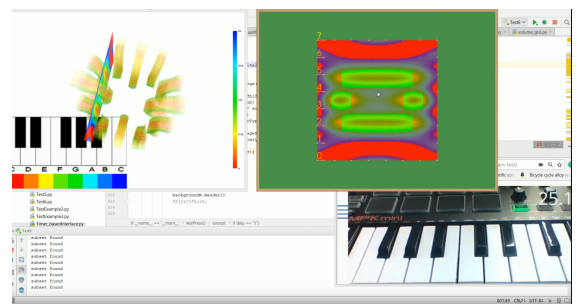

(b)

Figure 5: Interaction via Midi keyboard a) Music pattern definition with Midi Keyboard b) Highlighting of scalar field areas corresponding to defined pitch pattern

example of the physical applications were implemented in Python.

As a case study for our approach, we consider a superconductor field analysis. One of the particular geometric features of this field is so-called Abrikosov vortex [41] (Fig. 6 a), Fig. 7), which represents isosurfaces of the supercurrents as they circulate. The analysis of the vortex arrangements is applied to carry out judgements on the material properties and therefore an extra attention should be paid to the analysis of the superconductor scalar field. Below we discuss how our visual-auditory approach can be applied in this situation.

\subsection{Colour mapping quality}

In the general case, the scalar field contains a relatively large range of values. In the visual analysis, those values are interpreted as colours, which are not always easy to distinguish because of human colour perception. However, with a combination of a visual analysis with the auditory analysis we can highlight the regions of interest (ROI) by adjusting the visual mapping parameters (see Fig.6a and the accompanying video [42]).

An auditory approach can be potentially effective for very complex scalar fields with a big range of values as the mapping data one-, two-, or three-octave Cmaj. This range allows us to distinguish the field changes easier than with just a visual analysis and to track the colour mapping quality.

In order to be able to successfully distinguish the musical degrees within the scale, a listener should always keep in mind the first degree sound, which is the tonic, and compare all the other sounds to it. To help with this, the outside domain values are mapped into the tonic. Although such type of analysis is very similar to the procedures that are used by trained musicians and researchers, the entire procedure can be difficult for the untrained researcher.

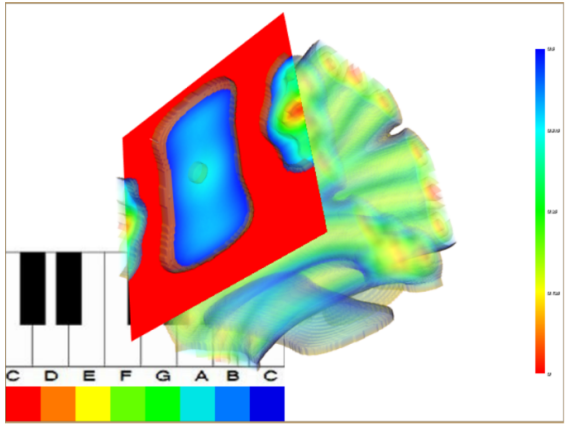

(a)

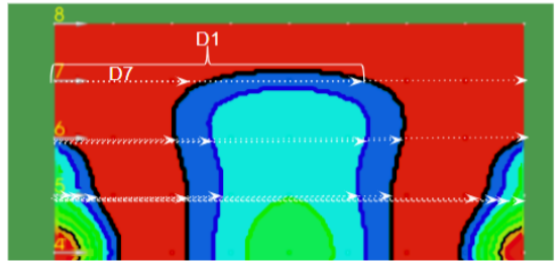

(b)

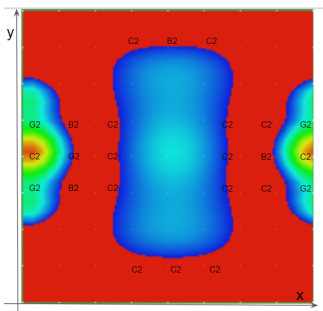

(c)

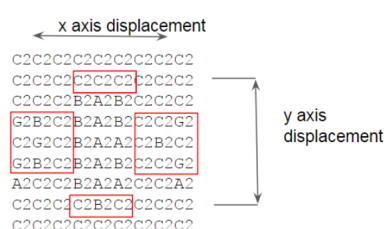

(d)
Figure 6: Examples of visual-auditory exploration of the second type superconductor field: a) The input scalar field with a 2D slice we analyse; the correspondent modal frequencies are denoted by colours and are presented in the form of a piano scale; b) MIDI field tracing with parallel ray casting. The modal areas are represented with the colours as in a); c) The field slice with the corresponding sound matrix representation (d) highlighting the displacements in the field.

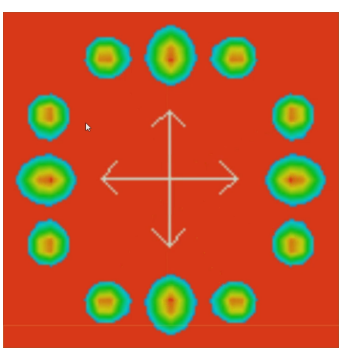

Figure 7: Field 2D slice with the highlighted vortex areas (adjustment of field ROI) and suggested directions for the auditory tracking of the field symmetrical features. 


\subsection{Symmetry and geometrical features changes tracking}

Another important application of our visual-auditory approach in the analysis of scalar fields for superconductors is symmetry and geometrical changes/features detection. For example, researchers in superconductors explore the issues of symmetry breaking and distortion in the vortex lattice or in the shape of the vortex itself [41]. The dynamic changes can be observed and analysed visually with scanning microscopy or with computer simulations. In our method, the small changes in symmetry and vortex geometry can easily be detected with the help of the sound (Fig. 6 c,d). In this example, we shoot two rays to the field areas that are supposed to be equal. The small differences in the field symmetry will sound like a non-perfect unison musical interval that can be aurally distinguished by the untrained researcher.

The main disadvantage of the colour mapping quality evaluation technique that was presented above is a finite sampling resolution of rays casting. The balance should be kept between the details users want to track and the speed of analysis. As a result of a small resolution, the analysis can take a relatively small time, but important features can be missed. One of the possible solutions is to specify ROI for an analysis and in combination with the guidance procedures. Potential ROIs and scanning directions can be identified automatically with the image processing techniques as it is done in the example shown in Fig. 6a and in the accompanying video [43]. For the superconductor analysis, this can be used to track the symmetrical features along the specified ray for a pair of the vortices or examine an area around a vortex to track its distortion.

\section{CONCLUSION AND FURTHER RESEARCH}

The main result of this research work is a unified theoretical and practical approach to visual-auditory Volume Rendering. The framework takes the scalar field as input and uses the ray-casting procedure that operates on some multisensory transfer function, to render it to the visual-auditory stimuli.

To introduce such an approach, we have taken the following steps. We have considered the colour mapping quality and tracking small scalar field features like symmetry break as two areas concerned with visual perception limitations. We have treated the scalar field and its domain as a representation of an object with optical and auditory properties that should be analysed and have suggested a HyperVolume model for such object representation.

We have discussed the similarities between visual-auditory properties modelling in order to address the problem of an efficient combination of visual-auditory stimuli. On base of those similarities we have proposed the visual-auditory mapping for two problems arising in area of visual analysis: the colour mapping quality and stacking small changes in symmetry of scalar field problems. The proposed mapping allows us to take most of the both sensory stimuli perceptional advantages and balance their disadvantages in the analysis process as they consider the models most close to physically based ones for optical and auditory properties modelling.

The light transfer based optical model or the model of the sound produced as a result of initial impulse propagation can provide not only a high level of realism but an intuitive way of the input parameters control in order to obtain the most realistic, desired result. These models, however, can be computationally expensive. For visualisation and computation purposes certain as- sumptions and adjustments are made to simplify the modelling in conventional Volume Rendering and the acoustic modelling.

Similarly to light, the sound propagation mechanism defines the perceivable characteristics: opacity and colour vs volume and pitch. Thus, the acoustic properties can also be rendered on the basis of the ray-marching procedure. We have considered the light and sound propagation similarities to introduce an approach to visual-auditory Volume Rendering. We have demonstrated how the auditory representation can be complementary to the visual one in gaining insight into the continuous scalar field in a case study of analysis of the scalar fields of superconductors. The proposed approach to visual-auditory analysis has been applied to some particular case studies. That has allowed us to judge on its advantages, limitations and possible adjustments.

Our experiments show possible limitations of the introduced method. As demonstrated in the colour mapping quality example, it may require some auditory skills from the user. We suggest the use of auditory analysis for the symmetrical data regions that allows for introducing the sound mappings which are easier to interpret.

Another limitation of our method is the sampling resolution. We overcome it through specifying the ROI for analysis and guidance procedures. This can be done automatically with the image processing (as demonstrated in the symmetry tracking example) or machine learning techniques, but currently out of the scope of this research. Finally, in this research, we have considered relatively simple scalar fields. However, the technique can be applied to more complex cases in such research areas as medical image analysis and molecular fields studies. Mapping the field and its additional derived features (gradient, curvature) to sound and an introduction of more complex mappings to music entities such as chords that are based on several rays shooting, are the areas of future research.

\section{REFERENCES}

[1] Y. Jung, J. Kim, A. Kumar, D. Feng, and M. Fulham, "Feature of interest-based direct volume rendering using contextual saliency-driven ray profile analysis," Computer Graphics Forum, vol. 37, no. 6, pp. 5-19, 2018.

[2] S. Djurcilov, K. Kim, P. Lermusiaux, and A. Pang, "Volume rendering data with uncertainty information," in Data Visualization 2001, D. S. Ebert, J. M. Favre, and R. Peikert, Eds. Vienna: Springer Vienna, 2001, pp. 243-252.

[3] D. A. Keim, F. Mansmann, J. Schneidewind, J. Thomas, and H. Ziegler, "Visual data mining," S. J. Simoff, M. H. Böhlen, and A. Mazeika, Eds. Berlin, Heidelberg: Springer-Verlag, 2008, ch. Visual Analytics: Scope and Challenges, pp. 7690.

[4] M. Ament, "Thesis: Computational visualization of scalar fields," 2014, accessed on 2017-11-12.

[5] P. Ljung, "Efficient methods for direct volume rendering of large data sets," Ph.D. dissertation, Linkoping University, Visual Information Technology and Applications, 2006.

[6] J. Kniss, G. Kindlmann, and C. Hansen, "Multidimensional transfer functions for interactive volume rendering," IEEE Transactions on Visualization and Computer Graphics, vol. 8, no. 3, pp. 270-285, July 2002. 
[7] C. Johnson, "Top scientific visualization research problems," IEEE Comput. Graph. Appl., vol. 24, no. 4, pp. 13-17, July 2004

[8] L. Patric, K. Jens, G. Eduard, H. Markus, H. C. D., and Y. Anders, "State of the art in transfer functions for direct volume rendering," Computer Graphics Forum, vol. 35, no. 3, pp. 669-691, 2016.

[9] G. Kramer, Auditory display: sonification, audification, and auditory interfaces, ser. Proceedings ; vol.18. Reading, Mass Wokingham: Addison-Wesley, 1994.

[10] T. Hermann, A. Hunt, and J. G. Neuhoff, Eds., The Sonification Handbook. Berlin, Germany: Logos Publishing House, 2011. [Online]. Available: http://sonification.de/ handbook

[11] Y. Allusse, P. Horain, A. Agarwal, and C. Saipriyadarshan, "Gpucv: A gpu-accelerated framework for image processing and computer vision," vol. 5359, 12 2008, pp. 430-439.

[12] M. Haidacher, S. Bruckner, A. Kanitsar, and M. E. Gröller, "Information-based transfer functions for multimodal visualization," in Proceedings of the First Eurographics Conference on Visual Computing for Biomedicine, ser. EG VCBM'08. Aire-la-Ville, Switzerland, Switzerland: Eurographics Association, 2008, pp. 101-108.

[13] M. Falk, I. Hotz, P. Ljung, D. Treanor, A. Ynnerman, and C. Lundstrom, "Transfer function design toolbox for fullcolor volume datasets," in 2017 IEEE Pacific Visualization Symposium (PacificVis), April 2017, pp. 171-179.

[14] S. Park and C. L. Bajaj, "Multi-dimensional transfer function design for scientific visualization," in ICVGIP, 2004.

[15] S. Fang, T. Biddlecome, and M. Tuceryan, "Imagebased transfer function design for data exploration in volume visualization,' in Proceedings Visualization '98 (Cat. No.98CB36276), Oct 1998, pp. 319-326.

[16] A. El Saddik, M. Orozco, M. Eid, and J. Cha, Haptics: General Principles, 1st ed. Springer Publishing Company, Incorporated, 08 2011, pp. 1-20.

[17] S. Bly, "Presenting information in sound," in Proceedings of the CHI '82 Conference on Human Factors in Computer Systems. ACM, 1982, pp. 371-375.

[18] E. Yeung, "Pattern recognition by audio representation of multivariate analytical data," Analytical Chemistry, vol. 52, no. 7, pp. 1120-1123, 1980.

[19] H. Kaper, E. Wiebel, and S. Tipei, "Data sonification and sound visualization," in Computing in Science and Engineering, vol. 1, no. 4, 1999, pp. 48-58.

[20] L. Gionfrida, A. Roginska, J. Keary, H. Mohanraj, and K. P. Friedman, "The triple tone sonification method to enhance the diagnosis of alzheimer's dementia," in The 22nd International Conference on Auditory Display (ICAD), 2016.

[21] H. Roodaki, N. Navab, A. Eslami, C. Stapleton, and N. Navab, "Sonifeye: Sonification of visual information using physical modeling sound synthesis," IEEE Transactions on Visualization and Computer Graphics, vol. 23, no. 11, pp. 2366-2371, Nov. 2017.

[22] R. Minghim and A. R. Forrest, "An illustrated analysis of sonification for scientific visualisation," in Proceedings Visualization '95, Oct 1995, pp. 110-117.
[23] S. K. Lodha, J. Beahan, T. Heppe, A. J. Joseph, and B. ZaneUlman, "Muse : A musical data sonification toolkit," 1997.

[24] L. Gionfrida and A. Roginska, "A novel sonification approach to support the diagnosis of alzheimer's dementia," Frontiers in Neurology, vol. 8, p. 647, 2017.

[25] F. Ribeiro, D. Florłncio, P. A. Chou, and Z. Zhang, "Auditory augmented reality: Object sonification for the visually impaired," in 2012 IEEE 14th International Workshop on Multimedia Signal Processing (MMSP), Sept 2012, pp. 319-324.

[26] J. T. Kajiya, "The rendering equation," SIGGRAPH Comput. Graph., vol. 20, no. 4, pp. 143-150, Aug. 1986.

[27] N. Max, "Optical models for direct volume rendering," IEEE Transactions on Visualization and Computer Graphics, vol. 1, no. 2, pp. 99-108, June 1995.

[28] M. Hadwiger, J. M. Kniss, C. Rezk-salama, D. Weiskopf, and K. Engel, Real-time Volume Graphics. Natick, MA, USA: A. K. Peters, Ltd., 2006.

[29] S. Roettger, S. Guthe, D. Weiskopf, T. Ertl, and W. Strasser, "Smart hardware-accelerated volume rendering," in Proceedings of the Symposium on Data Visualisation 2003, ser. VISSYM '03. Aire-la-Ville, Switzerland, Switzerland: Eurographics Association, 2003, pp. 231-238.

[30] S. G. Matt Pharr, Ambient Occlusion. Pearson Higher Education, 2004

[31] T. Takala and J. Hahn, "Sound rendering," in Proceedings of the 19th Annual Conference on Computer Graphics and Interactive Techniques, ser. SIGGRAPH ' $92 . \quad$ New York, NY, USA: ACM, 1992, pp. 211-220.

[32] S. Siltanen, T. Lokki, S. Kiminki, and L. Savioja, "The room acoustic rendering equation," vol. 122, p. 1624, 102007.

[33] P. R. Cook, Real Sound Synthesis for Interactive Applications. Natick, MA, USA: A. K. Peters, Ltd., 2002.

[34] A. Pasko, V. Adzhiev, B. Schmitt, and C. Schlick, "Constructive hypervolume modeling," Graph. Models, vol. 63, no. 6, pp. 413-442, Nov. 2001.

[35] R. Wu and G. Yu, "Improvements in hrtf dataset of 3d game audio application," in 2016 International Conference on Audio, Language and Image Processing (ICALIP), July 2016, pp. 185-190.

[36] M. Hewitt, Music Theory for Computer Musicians. Course Technology, CENGAGE Learning, 2008.

[37] "Midi keyboard interaction," 2019, password icad2019. [Online]. Available: https://vimeo.com/323545930

[38] W. Schroeder, K. Martin, and B. Lorensen, The Visualization Toolkit-An Object-Oriented Approach To 3D Graphics, 4th ed. Kitware, Inc., 2006.

[39] “Openal programmers guide," https://www.openal.org/ documentation/OpenAL_Programmers_Guide.pdf, 2007.

[40] “Opencv," http://opencv.org.

[41] H. Suderow, I. Guillamon, J. G. Rodrigo, and S. Vieira, "Imaging superconducting vortex cores and lattices with a scanning tunneling microscope," Superconductor Science and Technology, vol. 27, no. 6, p. 063001, 2014.

[42] “Colour quality check," 2019, password icad2019. [Online]. Available: https://vimeo.com/323547646

[43] "Scalar field symmetry analysis," 2019, password icad2019. [Online]. Available: https://vimeo.com/323547659 